\title{
Structural and magnetic properties of morphotropic phase boundary involved $\mathrm{Tb}_{1-\mathrm{x}} \mathrm{Gd}_{\mathrm{x}} \mathrm{Fe}_{2}$ compounds
}

\author{
Murtaza Adil ${ }^{1}$, Sen Yang ${ }^{1,(a)}$, Chao Zhou ${ }^{1,(b)}$, Khan Muhammad Tahir ${ }^{1}$, Ghani Awais ${ }^{1}$, \\ Fanghua Tian ${ }^{1}$, Jieqiong Wang ${ }^{1}$, Xiaoping Song ${ }^{1}$, Matthrew Suchomel ${ }^{2}$ AND Yang Ren ${ }^{2}$ \\ ${ }^{1}$ School of Science, Frontier Institute of Science and Technology, MOE Key Laboratory for \\ Nonequilibrium Synthesis and Modulation of Condensed Matter, State Key Laboratory for \\ Mechanical Behaviour of Materials, Xi'an Jiaotong University, Xi'an 710049, China \\ ${ }^{2}$ X-Ray Science Division, Argonne National Laboratory, Argonne, Illinions 60439, USA
}

\begin{abstract}
In the present paper, structural, magnetic and magnetostrictive properties of $\mathrm{Tb}_{1-\mathrm{x}} \mathrm{Gd}_{\mathrm{x}} \mathrm{Fe}_{2}(0 \leq \mathrm{x} \leq 1.0)$ were studied. Synchrotron $\mathrm{x}$-ray diffraction (XRD) results show the non-cubic symmetry of $\mathrm{Tb}_{1-\mathrm{x}} \mathrm{Gd}_{x} \mathrm{Fe}_{2}$ at room temperature and composition-induced crystallographic phase transition from rhombohedral phase to tetragonal phase. The Gd concentration dependent lattice parameters, lattice distortion and change of easy magnetic direction were detected by synchrotron XRD. With the Gd concentration increases, Curie temperature $\boldsymbol{T} \boldsymbol{c}$ increases while room temperature magnetization and magnetostriction coefficient $\lambda_{111}$ and the anisotropy of $\mathrm{TbFe}_{2}$ decrease. The decrease in spontaneous magnetostriction coefficient $\lambda_{111}$ with increasing Gd substitution can be understood on the basis of the single-ion model; the corresponding decrease of magnetostriction for $\mathrm{Tb}_{1-\mathrm{x}} \mathrm{Gd}_{\mathrm{x}} \mathrm{Fe}_{2}$, and the large magnetostriction value occurs on the Tb-rich side, are ascribed to decrease of $\lambda_{111}$.
\end{abstract}

Key Words: Magnetic phase boundary, Synchrotron X-ray diffraction, Lattice distortion, Magnetostriction

\footnotetext{
${ }^{(a)}$ E-mail: yang.sen@mail.xjtu.edu.cn

${ }^{(b)}$ E-mail: chaozhou.xjtu@gmail.com
} 


\section{Introduction}

For more than half of a century the ferroelectric morphotropic phase boundary (MPB), a phase boundary separating two ferroelectric phases of different crystallographic symmetries in the composition temperature phase diagram [1-3], has drawn constant interest due to large functional response at compositions in the vicinity of MPB. Ferromagnets systems are physically parallel to ferroelectrics [4] with contrast in functionalities and experimental phenomena, i.e polarization in a ferroelectric is a direct consequence of the local atomic displacement in the lattice while the magnetic moment created in a ferromagnet is the result of spin-orbit coupling [5]. In both systems, the order parameters are coupled to the lattice, leading to piezoelectric and magnetoelastic effect, respectively [6]. Thus, it follows naturally that hybrid MPBs between different types of ferroics exist [7], resulting in the enhancement of the magnetoelastic properties of ferromagnets.

Recently, Yang et al. reported a magnetic MPB in the $\mathrm{TbCo}_{2}-\mathrm{DyCo}_{2}$ system, proposing that MPB in ferromagnets can lead to large magnetostriction. Although its MPB composition shows enhanced magnetostriction, saturation magnetization and magnetic susceptibility, the Curie temperature $\boldsymbol{T}_{\boldsymbol{c}}$ far below room temperature in this system limits its wide applications [8]. Zhou et al. reported an MPB in $\mathrm{Tb}_{1-\mathrm{x}} \mathrm{Gd}_{\mathrm{x}} \mathrm{Co}_{2}$ system; it shows greatly weakened magnetostriction (near zero) at MPB composition (called inverse effect of MPB on magnetostriction) [9]. In our previous work, we have shown a newly discovered type of MPB in the ferromagnetic $\mathrm{Tb}_{1-\mathrm{x}} \mathrm{Gd}_{\mathrm{x}} \mathrm{Fe}_{2}$ system exhibiting low magnetostriction at MPB composition $\mathrm{x}=0.9$ [10]. It is important to investigate the cause of low magnetostriction at MPB composition. Cullen and Clark [11] showed that in $\mathrm{RFe}_{2}$ Laves compounds possessing spontaneous axis of magnetization parallel to the [111] direction, an internal rhombohedral distortion takes place. This distortion is coupled to the external strain and leads to the giant observed magnetostriction. As MPB corresponds to the coexistence of rhombohedral and tetragonal phases, magnetostriction at MPB would be contributed by both rhombohedral $\left(\lambda_{111}\right)$ and tetragonal $\left(\lambda_{100}\right)$ distortions. In the present work, we calculate the values of $\lambda_{111}$ and $\lambda_{100}$ 
from the synchrotron x-ray diffractometer (XRD) measurements on polycrystalline samples. Because $\lambda_{111}$ counts mainly for the measured magnetostriction, and the decrease of $\lambda_{111}$ exceeds the increase of $\lambda_{100}$ with $\mathrm{Gd}$ concentration increases, the decrease of the magnetostriction in polycrystalline $\mathrm{Tb}_{1-\mathrm{x}} \mathrm{Gd}_{x} \mathrm{Fe}_{2}$ compounds is observed. We ascribe the observed phenomena in the MPB composition to the physical relation between the single-ion model and the spontaneous magnetostriction coefficient.

\section{Experimental details}

The ingots of $\mathrm{Tb}_{1-\mathrm{x}} \mathrm{Gd}_{\mathrm{x}} \mathrm{Fe}_{2}(0 \leq \mathrm{x} \leq 1)$ were prepared by arc melting $\mathrm{Fe}$ (of purity $99.9 \mathrm{wt} \%$ ), $\mathrm{Tb}(99.95 \mathrm{wt} \%)$ and $\mathrm{Gd}(99.95 \mathrm{wt} \%)$ under a purified argon atmosphere. The proportions of the rare earths were greater than the stoichiometric amount by $0.5 \mathrm{wt} \%$ in order to compensate for loss from oxidation during melting. The as-cast samples were annealed in sealed quartz tube at $850 \mathrm{~K}$ for three days. In order to determine the crystal symmetry, highresolution synchrotron XRD experiments were performed with samples grounded into powders and sealed into quartz capillaries with a diameter of $0.3 \mathrm{~mm}$ at beamline 11-BM-B (with a strain resolution of about $5 \times 10^{-4}$ ), Advanced Photon Source (APS), Argonne National Laboratory. During the synchrotron XRD measurement, the capillary was rotating in order to average the intensity as well as to reduce the preferred orientation effect. The wavelength of

synchrotron x-ray was $0.413677 \AA$. Curie temperatures $\boldsymbol{T}_{\boldsymbol{c}}$ from the $\boldsymbol{M}$ - $\boldsymbol{T}$ (temperature dependence of magnetization) curves were measured with the vibrating sample magnetometer (VSM) under magnetic field of 500 Oe. The room temperature saturation magnetization $\boldsymbol{M}_{\boldsymbol{s}}$ was measured by superconducting quantum interference device (SQUID) in magnetic field up to $40 \mathrm{kOe}$. Standard strain-gauge technique has been applied to measure magnetostriction $(\varepsilon)$ at room temperature under magnetic field of $50 \mathrm{kOe}$.

\section{Results}


The typical synchrotron X-ray diffraction patterns for $\mathrm{Tb}_{1-\mathrm{x}} \mathrm{Gd}_{\mathrm{x}} \mathrm{Fe}_{2}(\mathrm{x}=0,0.5$ and 1.0) are shown in Fig.1(a) several well defined diffraction peaks appearing at $2 \theta=5.49^{\circ}, 9.02^{\circ}$, $10.62^{\circ}, 11.08^{\circ}, 13.91^{\circ}, 15.71^{\circ}, 16.65^{\circ}, 18.15^{\circ}, 19.01^{\circ}, 20.36^{\circ}, 21.09^{\circ}$ and $25.18^{\circ}$ can be assigned as (111), (220), (311), (222), (331), (422), (511), (440), (531), (620), (731), and (800) respectively. Figure 1(b) shows the splitting of (222), (440) and (800) reflections measured by synchrotron XRD. It is clearly seen that for the compositions of $x=0,0.5,0.8$, the characteristic reflections of (222) and (440) split into two peaks but (800) reflection has no splitting. These features characterize a rhombohedral structure for ferromagnetic $\mathrm{Tb}_{1}$ ${ }_{x} \mathrm{Gd}_{x} \mathrm{Fe}_{2}$ with easy magnetization direction along [111] (Fig.1(d)), and the corresponding lattice parameters are shown in Fig. 1(c). The splitting of (222) and (440) reflections decreases with increasing Gd content, which is indicative of a decrease of lattice distortion and spontaneous magnetostriction of the $\mathrm{Tb}_{1-\mathrm{x}} \mathrm{Gd}_{\mathrm{x}} \mathrm{Fe}_{2}$ compounds.

For $\mathrm{x}=1$, no splitting in (222) and (440) reflections is observed but only characteristic splitting in (800) peak. These features show that the ferromagnetic $\mathrm{GdFe}_{2}$ has a lower symmetry than cubic and characterize a tetragonal symmetry for $\mathrm{GdFe}_{2}$, and the corresponding lattice parameters are shown in Fig.2. The direction of the spontaneous magnetization $\boldsymbol{M}_{\boldsymbol{s}}$ is along [100] (Fig.1(d)). As for the XRD profile of 0.9Gd, it corresponds to the superposition of the $\boldsymbol{R}$ symmetry profile and $\boldsymbol{T}$ symmetry profile - MPB structure we have shown in previous work [10].

In the past it was shown that during ferromagnetic transition the crystal structure remains unchanged i.e as paramagnet cubic changed into ferromagnetic cubic below the Curie temperature. However, high resolution synchrotron $\mathrm{x}$-ray diffractometry $\mathrm{XRD}$, is a direct evidence for lower symmetry of cubic ferromagnets. These results reveal that ferromagnetic transition is also a structural transition, resulting a low crystallographic symmetry that follows to the spontaneous magnetization $M_{S}$ direction is a general effect for all cubic ferromagnets. $[8,9,10]$ XRD profiles for $\mathrm{Tb}_{1-\mathrm{x}} \mathrm{Gd}_{\mathrm{x}} \mathrm{Fe}_{2}$ system exhibit a composition-induced crystallographic phase transition from rhombedral phase ( $\boldsymbol{R}$-symmetry) to tetragonal phase ( $\boldsymbol{T}$-symmetry) with increasing Gd concentration. The observed structural evolution at room temperature (298K) is 
consistent with the established relation between the easy axis direction and crystal symmetry as shown in Figs.1(b, c) [12]. This composition-induced crystallographic phase transition considered as "spin-reorientation transition (SRT)" when the rotation of magnetic moment occurs at ferro-ferro transition. $[13,14]$ Therefore, phase boundary between the rhombedral phase ( $\boldsymbol{R}$-symmetry) to tetragonal phase ( $\boldsymbol{T}$-symmetry) is a ferromagnetic phase boundary as well as crystallographic phase boundary, so we can say that the morphotropic phase boundaries in ferromagnetics systems are magnetic morphotropic phase boundaries (MMPB). These MMPB's are similar to the ferroelectric MPB's. [1-3]

Fig.1(c) shows that the composition dependence of lattice parameters; it is seen that the system possesses $\boldsymbol{R}$ symmetry when x is below 0.9, coexistence of $\boldsymbol{R}$ and $\boldsymbol{T}$ phases (at MPB) when $\mathrm{x}=0.9$, and $\boldsymbol{T}$ symmetry when $\mathrm{x}>0.9 . \boldsymbol{R}$-phase lattice parameters $\boldsymbol{a}_{\boldsymbol{R}}$ and $\boldsymbol{\alpha}_{\boldsymbol{R}}$ increase with increasing Gd concentration, since Gd has a larger ionic radius than Tb. For $\boldsymbol{T}$-Phase lattice parameter $\boldsymbol{a}_{T}$ is little bit larger than $\mathbf{c}_{\boldsymbol{T}}$, indicating small distortion at $\boldsymbol{T}$-phase. The yellow shaded area corresponds to a mixture state of $\boldsymbol{R}$-phase and $\boldsymbol{T}$-phase [10]. The calculated lattice parameters for $\mathrm{TbFe}_{2}$ and $\mathrm{GdFe}_{2}$ are $7.341 \AA$ and $7.390 \AA$ respectively, similar to the published values $[15,16]$.

The $\boldsymbol{M - T}$ curves for $\mathrm{Tb}_{1-\mathrm{x}} \mathrm{Gd}_{\mathrm{x}} \mathrm{Fe}_{2}(\mathrm{x}=0,0.2,0.4,0.6,0.8$ and1.0) are shown in Fig.2(a). It can be seen that $\boldsymbol{T}_{\boldsymbol{c}}$ increases from $710 \mathrm{~K}$ to $798 \mathrm{~K}$ with increasing Gd content. In rare-earthtransition-metal $\left(\mathrm{RFe}_{2}\right)$ compounds, $\boldsymbol{T}_{\boldsymbol{c}}$ is determined by the $3 d-3 d, 3 d-4 f$, and $4 f-4 f$ exchange interactions because of localized $4 f$ electrons of the rare earth. Since the spin-orbit coupling almost vanishes due to the isotropy of $\mathrm{Gd}$, we only concentrate on the exchange interaction in the absence of any crystal field effect (CFE). [17] As the $4 \mathrm{f}$ orbital of Gd is half filled and $5 \mathrm{~d}$ orbital less than half-filled and the $3 \mathrm{~d}$ states Fe are filled more than half, by considering the ferromagnetic exchange interaction between the $4 \mathrm{f}$ and $5 \mathrm{~d}$ spins and inter-atomic exchange interactions between the $3 \mathrm{~d}$ and $5 \mathrm{~d}$ electrons leading to an antiparallel $4 \mathrm{f}-3 \mathrm{~d}$ coupling. $[18,19]$ As Gd substitutes for $\mathrm{Tb}$ in $\mathrm{Tb}_{1-\mathrm{x}} \mathrm{Gd}_{\mathrm{x}} \mathrm{Fe}_{2}$, some of $\mathrm{Fe}-\mathrm{Fe}$ or $\mathrm{Tb}-\mathrm{Gd}$ and $\mathrm{Tb}-\mathrm{Fe}$ interactions are replaced by $\mathrm{Gd}-\mathrm{Fe}$ interactions. This $\mathrm{Gd}-\mathrm{Fe}$ hybridization is strong in $\mathrm{GdFe}_{2}$ [20]. Therefore, it is coherent that $T_{c}$ increases with increasing $G d$ content in $\operatorname{Tb}_{1-x} \mathrm{Gd}_{x} \mathrm{Fe}_{2}$ alloys. Extrapolating 
the line to $\mathrm{x}=0$, the Curie temperature for $\mathrm{TbFe}_{2}$ is $710 \mathrm{~K}$, which is slightly higher than $697 \mathrm{~K}$ reported earlier for $\mathrm{TbFe}_{2}[21]$. As $\boldsymbol{T}_{\boldsymbol{C}}$ is determined by $3 \mathrm{~d}-4 \mathrm{f}$ exchange interactions of which the values vary in $\mathrm{TbFe}_{2}$ and $\mathrm{GdFe}_{2}$, the composition dependence of $\boldsymbol{T}_{\boldsymbol{C}}$ in Fig. 2(c) is not linear. $\mathrm{GdFe}_{2}$ is free of crystal field effects since $\mathrm{Gd}^{3+}$ is in an $S$ state. Hence assuming ferromagnetic coupling the $\mathrm{Fe}$ moment in $\mathrm{GdFe}_{2}$ is $1.62 \mu_{\mathrm{B}}$ as contrasted with $1.45 \mu_{\mathrm{B}}$ in $\mathrm{LuFe}_{2}$ or $\mathrm{ZrFe}_{2}$. Thus, the iron moment is variable in the $\mathrm{LnFe}_{2}$ series [22] and the, $\mathrm{RE}-\mathrm{Fe}$ or $\mathrm{Fe}-\mathrm{Fe}$ exchange interaction is the most important interaction that determines $\boldsymbol{T}_{\boldsymbol{c}}$ in these compounds [23].

The magnetic field dependence of the magnetization for the $\mathrm{Tb}_{1-\mathrm{x}} \mathrm{Gd}_{\mathrm{x}} \mathrm{Fe}_{2}$ alloys at room temperature is shown in Fig.2(b). It is noted that the magnetization of the alloys is close to saturation at the field of $40 \mathrm{kOe}$. The saturation magnetizations $\boldsymbol{M}_{\boldsymbol{s}}$ of $\mathrm{Tb}_{1-\mathrm{x}} \mathrm{Gd}_{\mathrm{x}} \mathrm{Fe}_{2}$ compounds as a function of the Gd concentration are shown in Fig. 2(c). It is found that $\boldsymbol{M}_{\boldsymbol{s}}$ decreases with increasing Gd concentration. Since the moment of $\mathrm{Gd}$ is antiparallel to $\mathrm{Fe}$ and parallel to $\mathrm{Tb}$, the observed magnetic moment $\mu_{S}$ of $\mathrm{Tb}_{1-\mathrm{x}} \mathrm{Gd}_{\mathrm{x}} \mathrm{Fe}_{2}$ can be expressed as

$$
\mu_{s}=(1-x) \mu_{T b}+x \mu_{G d}-2 \mu_{F e}
$$

Assuming that $\mathrm{Tb}$ and $\mathrm{Gd}$ as free ions, their calculated magnetic moments are $=6.92 \mu \mathrm{B}$ and $=5.3 \mu \mathrm{B}$ respectively. Moreover, $\boldsymbol{M}_{\boldsymbol{s}}$ value for $\mathrm{TbFe}_{2}$ is $80.77 \mathrm{emu} / \mathrm{g}$ and for $\mathrm{GdFe}_{2}$ is $54.65 \mathrm{emu} / \mathrm{g}$, which are equivalent to $3.91 \mu_{\mathrm{B}}$ and $2.63 \mu_{\mathrm{B}}$ respectively. Subtracting these values from free ion values of $\mathrm{Tb}$ and $\mathrm{Gd}$, one can calculate magnetic moment per iron is $2.5 \mu_{\mathrm{B}}$ for $\mathrm{TbFe}_{2}$ and $2.1 \mu_{\mathrm{B}}$ for $\mathrm{GdFe}_{2}$. Previous work shows that magnetic moment per iron is $1.64 \mu_{\mathrm{B}}$ and $1.60 \mu_{\mathrm{B}}$ for $\mathrm{TbFe}_{2}$ and $\mathrm{GdFe}_{2}$ respectively. [24, 25] Our calculated values agree with previously reported values of $\mu_{F e}$ equal to $2.1 \mu_{\mathrm{B}}$ for $\mathrm{TbFe}_{2}$ and $\mathrm{GdFe}_{2}$ [26], it shows that in $\mathrm{RFe}_{2}$ compound a higher field is required to measure saturation magnetization otherwise $\mu_{F e}$ will be larger. At room temperature, the magnetostriction ( $\varepsilon$ ) of the $\mathrm{Tb}_{1-\mathrm{x}} \mathrm{Gd}_{\mathrm{x}} \mathrm{Fe}_{2}$ under field of $50 \mathrm{kOe}$ is shown in Fig.3. The magnetostrictions for all samples are not saturated until the field reaches $50 \mathrm{kOe}$. The magnetostriction $(\varepsilon)$ is strongly dependent on the magnetic 
anisotropy and the contribution of the Gd to the magnetocrystalline anisotropy of the rareearth site. Fig. 3 shows that magnetostriction gradually decreases with the substitution of $\mathrm{Gd}$ for $\mathrm{Tb}$ under the same field for Gd concentration $\mathrm{x}$ from 0 to 1.0 . It is obvious that $\mathrm{TbFe}_{2}$ possesses a much larger magnetostriction than $\mathrm{GdFe}_{2}$. This indicates that the $\mathrm{Gd}$ ion has a lower anisotropy than the $\mathrm{Tb}$ ion because the rare-earth sublattices control magnetostrictive properties.

In analogy with the behavior of saturated magnetization of this alloy, its saturated magnetostriction also decreases. It can be explained by single-ion model; the saturation magnetostriction is proportional to $M_{S R}^{3}$, where $M_{S R}$ is the saturation magnetization of the rare-earth sublattice [27]. Since the average of $M_{S R}$ is decreasing with Gd content, the magnetostriction also decreases.

\section{Discussion}

The rare earth-iron $\left(\mathrm{REF}_{2}\right)$ Laves phase compounds are the well-known compounds possessing huge magnetostrictions $\left(=2000 \times 10^{-6}\right)$ at room temperature. It is surprising that the magnetostriction arises almost exclusively from the lattice distortion. This distortion lowers the symmetry and drives an external rhombohedral distortion $\left(\lambda_{111}\right)[28,29]$. Thus, the magnetostriction coefficient $\lambda_{111}$ is of utmost importance, and it implies the magnetostrictive potential of the compound because $\lambda_{111} \gg \lambda_{100}$ in $\mathrm{RFe}_{2}$. It is widely accepted that the twofold splitting of the (440) reflection is related to a rhombohedral distortion and tetragonal distortion of the cubic lattice by $\lambda_{111}=\Delta \alpha$ and $\lambda_{100}=(2 / 3) \Delta \alpha /$ a, respectively. Here, $\Delta \alpha$ is a deviation of the angle between neighboring edges of the distorted cube from $\pi / 2$ and $\Delta a$ is the difference between edges of the distorted cube [30,31]. Careful analysis of the diffraction profiles of the rhombohedrally distorted cube structure $(R-3 m)$ allows a straight forward determination of $\lambda_{111}[32,33]$. In particular, for ( $\left.h h h\right)$-type and (hh0)-type cubic reflections, the splitting $\Delta d / d$ equals $3 / 4 \lambda_{111}$ and $\lambda_{111}$, respectively [34]. In the present study we used splitting of (222) and (800) reflections to determine $\lambda_{111}$ and $\lambda_{100}$ for $\operatorname{Tb}_{1-\mathrm{x}} \mathrm{Gd}_{\mathrm{x}} \mathrm{Fe}_{2}$. The calculated spontaneous magnetostriction coefficients $\lambda_{111}$ and $\lambda_{100}$ are shown in Fig.4(a), 
where the dashed dot lines are guides to the eyes. With the increase of Gd concentration, the $\lambda_{111}$ decreases to almost zero for $\mathrm{GdFe}_{2}$ with hard easy magnetisation direction (EMD) along [111], and $\lambda_{100}$ shows minimum for $\mathrm{TbFe}_{2}$ with hard EMD along [100]. For $\mathrm{TbFe}_{2}$ and $\mathrm{GdFe}_{2}$ the calculated values of $\lambda_{111}$ and $\lambda_{100}$ are $2391 \times 10^{6}$ and $45 \times 10^{-6}$, these results agree with previous data $[35,37]$.

Previous work shows that in $\mathrm{R}_{1-\mathrm{x}} \mathrm{M}_{\mathrm{x}} \mathrm{Fe}_{2}$ ( $\mathrm{R}=$ rare earth, $\mathrm{M}=$ transition elements) the easy magnetization direction of the mixed compound is the same as that of the predominant rare earth [30,37]. Therefore, for the Tb-rich side $(x<0.9)$, the rhombohedral distortion dominates, whereas for the Gd-rich side $(x>0.9)$, the tetragonal distortion prevails as shown in Figs.1(b,c). For Tb-rich side the splitting of (222) and (440) reflections are mainly contributed from the rhombohedral distortion. The easy direction of $\mathrm{TbFe}_{2}$ is parallel to [111] the calculated distortion is 0.0024 , corresponding to a rhombohedral angle $\alpha_{R}=89.85^{\circ}$. This result shows a good agreement with single crystal data [37] and previous x-ray study [38]. With the increase of Gd concentration the rhombohedral distortion becomes less and vanishes at the Gd-rich side. Under this consideration, for the Gd-rich compounds $(x>0.9)$, the tetragonal distortion results in the splitting of (800) reflections. For polycrystalline compounds the saturation magnetostriction can be obtained by the following relation:

$$
\lambda_{s}=3 / 5 \lambda_{111}+2 / 5 \lambda_{100}
$$

Calculated and experimental values show that saturation magnetostriction decreases monotonously with increasing Gd content (Fig.4(b)). The increase of $\lambda_{100}$ was not reflected in $\lambda s$, which may be attributed to the gradual decrease of $\lambda_{111}$.

According to the single-ion model the spontaneous magnetostriction $\lambda_{111}$ varies with temperature as $M^{3} R(T)$. Assuming the $\boldsymbol{R}$ sublattice moments decrease with increasing Curie temperature, the increase in $\boldsymbol{T}_{c}$ with increasing Gd content results in a decrease of $M_{R}(T)$ at room temperature, which in turn leads to a reduction of the spontaneous magnetostriction. Since the $\boldsymbol{T}_{\boldsymbol{c}}$ gradual increase due to Gd substitution so it is reasonable that the $\lambda_{111}$ change as one can see from Fig. 4(a). However, single-ion model is unable to explain the change of $\lambda_{100}$ 
due to Gd substitution. According the structural distortion model proposed by Clark et al. [35], only transition metals contribute to $\lambda_{100}$. A plausible theory of anisotropic magnetostriction in the band-magnetism model, capable of describing the transition metal sublattice in $\mathrm{RFe}_{2}$, has not yet been developed. A further study is required to explain this mechanism.

\section{Conclusion}

The structural, magnetic, and magnetostrictive properties of $\mathrm{Tb}_{1-\mathrm{x}} \mathrm{Gd}_{\mathrm{x}} \mathrm{Fe}_{2}(0 \leq \mathrm{x} \leq 1)$ alloys have been investigated. The increase of Curie temperature with increasing Gd concentration can be attributed to the increasing strength of $3 d-4 f$ coupling. The Gd substitution in $\mathrm{Tb}_{1-}$ ${ }_{x} \mathrm{Gd}_{\mathrm{x}} \mathrm{Fe}_{2}$ decreases the saturation magnetization $\boldsymbol{M}_{\boldsymbol{s}}$, which is ascribed to the parallel moments between $\mathrm{Tb}$ and $\mathrm{Gd}$. The decrease in magnetostriction coefficient $\lambda_{111}$ may cause to the decrease of magnetostriction of $\mathrm{Tb}_{1-\mathrm{x}} \mathrm{Gd}_{\mathrm{x}} \mathrm{Fe}_{2}$ alloys with increasing Gd Substitution. The anisotropy of $\mathrm{TbFe}_{2}$ decreases as increasing Gd content and the optimal magnetostriction occurs at the Tb-rich side for $\mathrm{Tb}_{1-\mathrm{x}} \mathrm{Gd}_{\mathrm{x}} \mathrm{Fe}_{2}$. Furthermore, magnetostriction behavior of $\mathrm{Tb}_{1-}$ ${ }_{x} \mathrm{Gd}_{\mathrm{x}} \mathrm{Fe}_{2}$ can be understood based on single-ion model.

\section{Acknowledgement}

This work was supported by the National Basic Research Program of China (Grant No. 2012CB619401), National Natural Science Foundation of China (Grant Nos. 51222104, 51371134, 51471125, and 51431007), Program for Key Science and Technology Innovative Team of Shaanxi Province (No. 2013KCT-05), and Collaborative Innovation Center of Suzhou Nano Science and Technology. Use of the Advanced Photon Source at Argonne National Laboratory was supported by the U.S. Department of Energy (DE-AC0206CH11357). 


\section{Figure Captions}

Figure 1. Fig 1 (a). Full synchrotron x-ray diffraction patterns of $0,0.5$ and $1.0 \mathrm{Gd}$ (with wavelength of $0.413677 \mathrm{Ang}$ ). (b) Typical 222, 440 and 800 peaks, of 0, 0.5, 0.8, 0.9, and 1.0 Gd. Bold black, blue and red line are experimental data, thin red and blue lines are fitted one (c) Composition dependence of lattic parameters $\left(a_{R}\right.$ and $\alpha_{R}$ stand for the lattice parameters of $\boldsymbol{R}$-phase, $\mathrm{a}_{\mathrm{T}}$ and $\mathrm{c}_{\mathrm{T}}$ for that of the $\boldsymbol{T}$-phase. The yellow shaded area corresponds to a 2-phase mixture of rhombohedral and tetragonal phases. (d) Rhombohedral and tetragonal structures.

Figure 2. Fig. 2. (a) $\boldsymbol{M}$ - $\boldsymbol{T}$ curves (b) $\boldsymbol{M}-\boldsymbol{H}$ hysteresis loops (c) composition dependend Curie temperature and saturation magnetisation for $\mathrm{Tb}_{1-\mathrm{x}} \mathrm{Gd}_{\mathrm{x}} \mathrm{Fe}_{2}(\mathrm{x}=0,0.2,0.4,0.8,0.9,1.0)$ alloy at $298 \mathrm{~K}$.

Figure 3. Fig.3 Magnetostriction ( $\varepsilon$ ) for polycrystalline $\mathrm{Tb}_{1-\mathrm{x}} \mathrm{Gd}_{\mathrm{x}} \mathrm{Fe}_{2}$ alloys at room temperature under field of $50 \mathrm{kOe}$.

Figure 4. Fig 4. (a) Composition dependent magnetostriction coefficients $\lambda_{111}$ and $\lambda_{100}$ (The dash lines are guides to eyes) (b) Calculated and Experimental and saturation magnetostriction $\lambda \mathrm{s}$ for $\mathrm{Tb}_{1-\mathrm{x}} \mathrm{Gd}_{\mathrm{x}} \mathrm{Fe}_{2}(\mathrm{x}=0,0.5,0.7,0.8,0.9$ and 1.0). 
Figure 1 : synchrotron $\mathrm{x}$-ray diffraction patterns

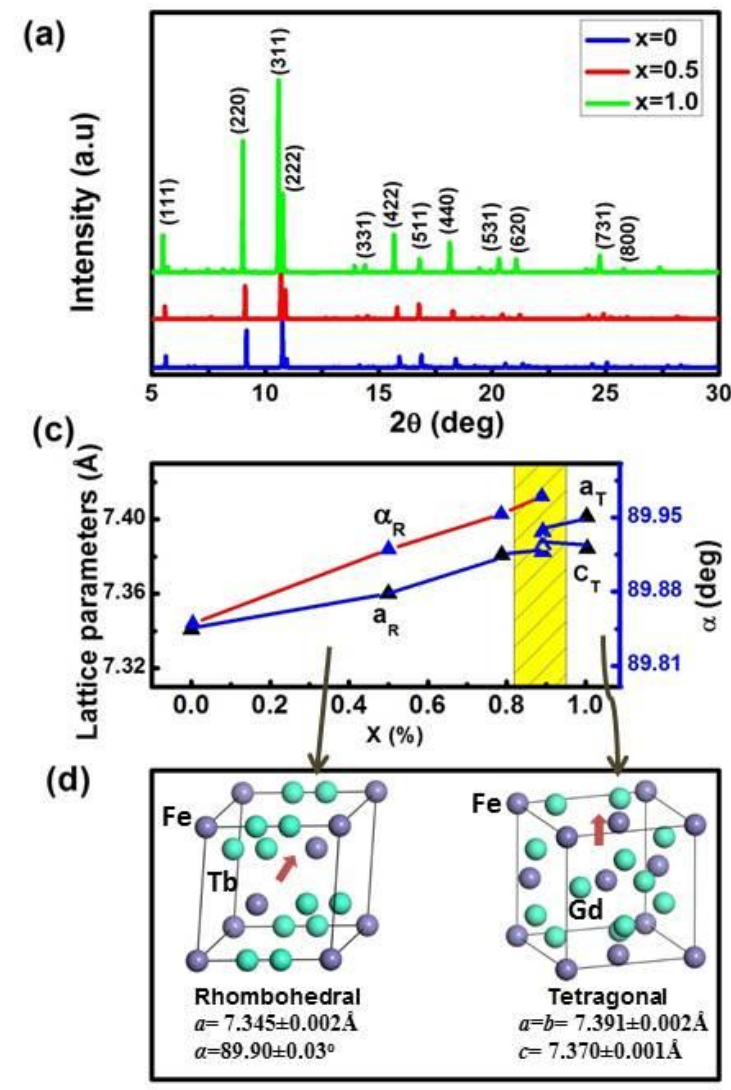

(b)

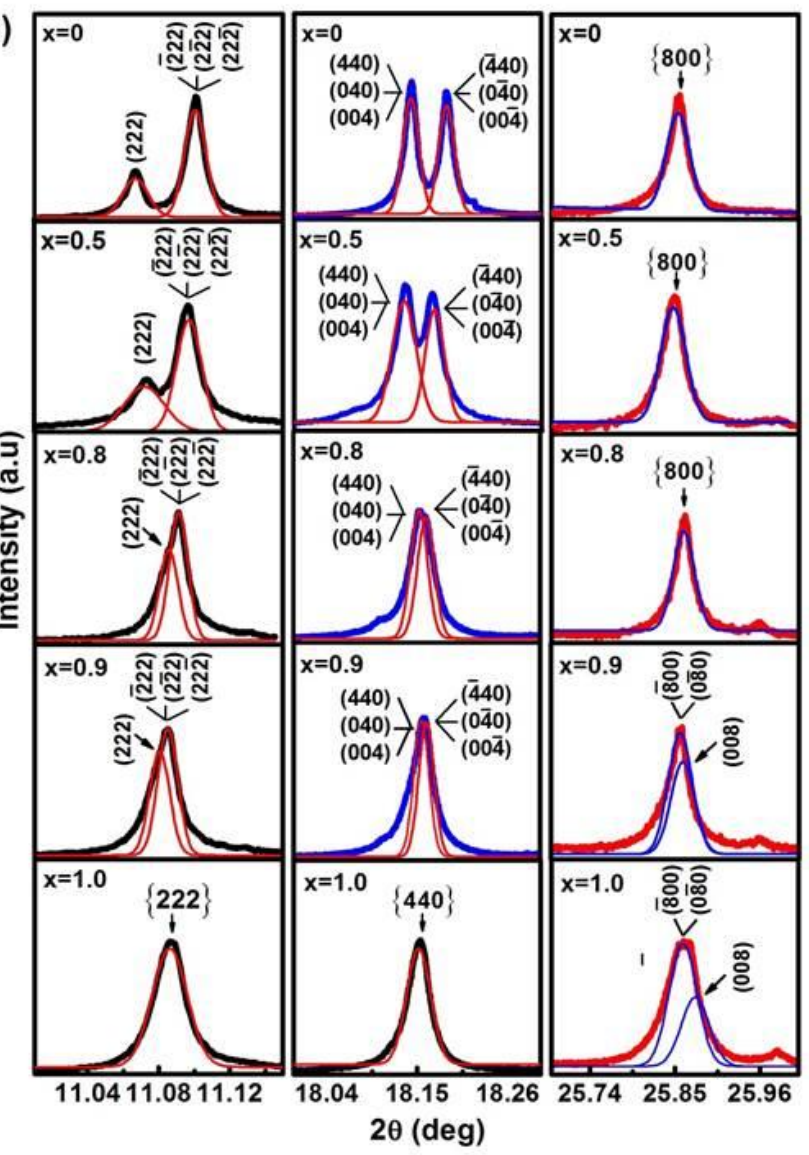


Figure 2: $\boldsymbol{M}-\boldsymbol{T}$ curves, $\boldsymbol{M}-\boldsymbol{H}$ hysteresis loops, composition dependend Curie temperature and saturation magnetisation
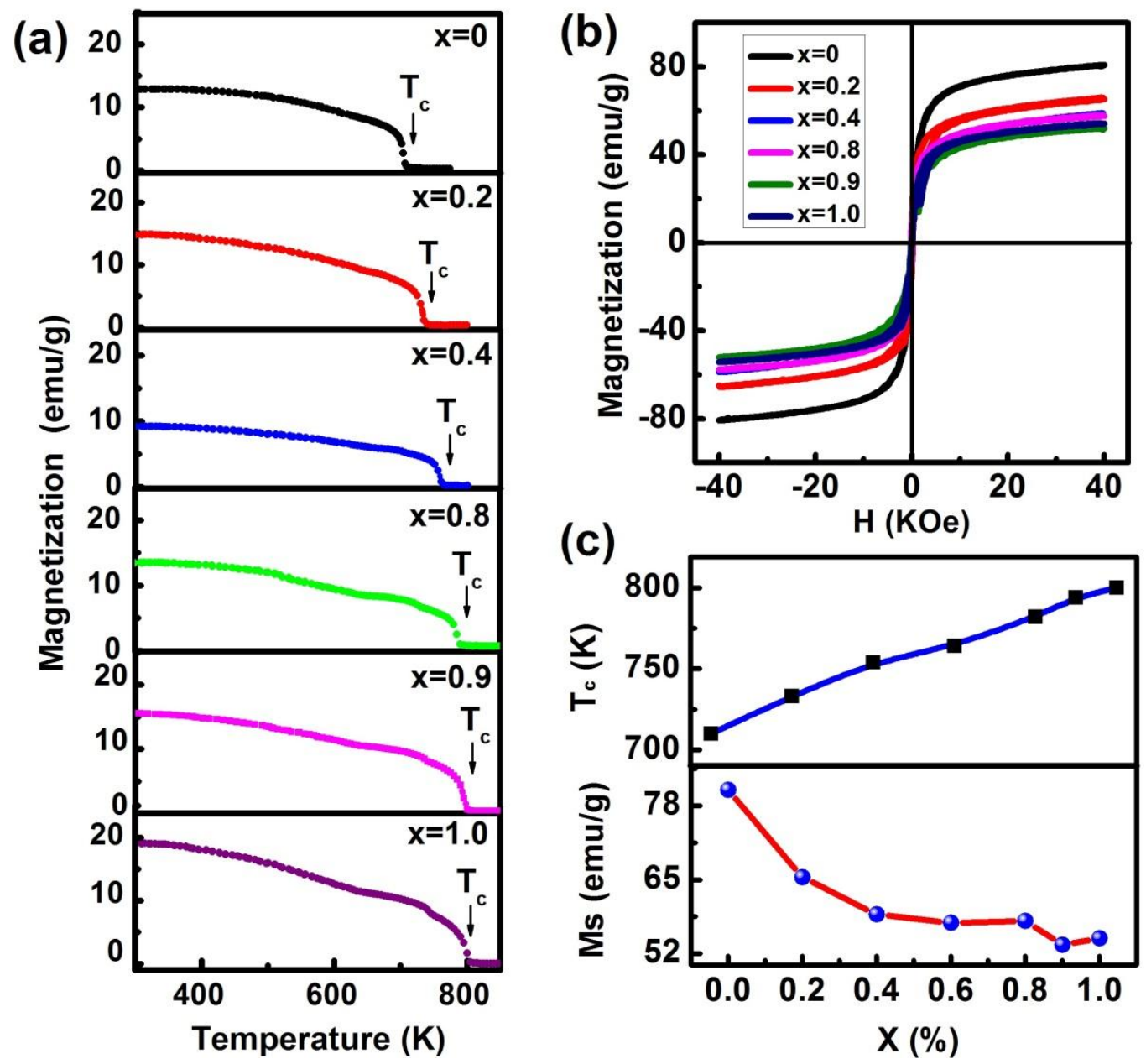
Figure 3: Magnetostriction
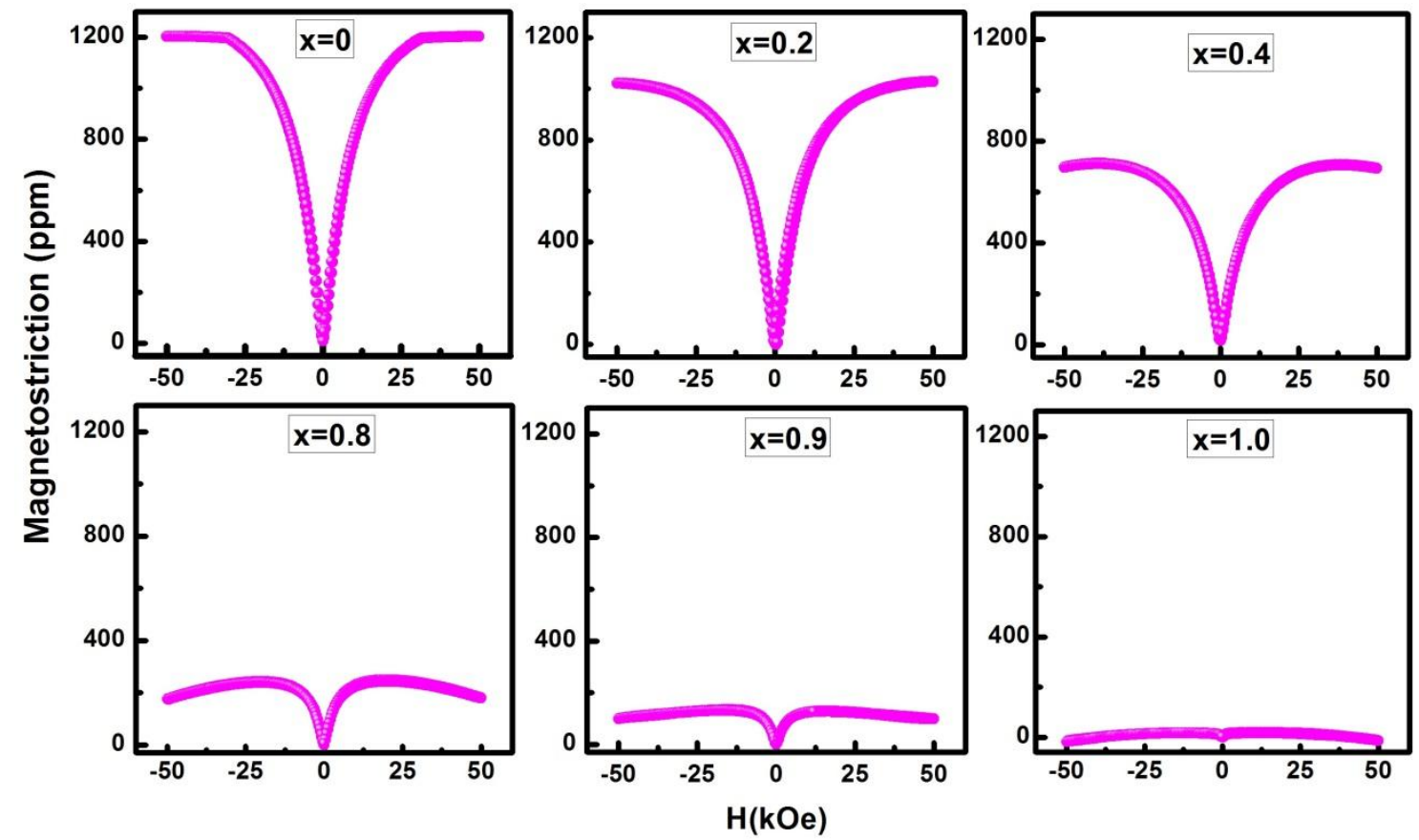
Figure 4: Composition dependent magnetostriction coefficients $\lambda_{111}$ and $\lambda_{100}$
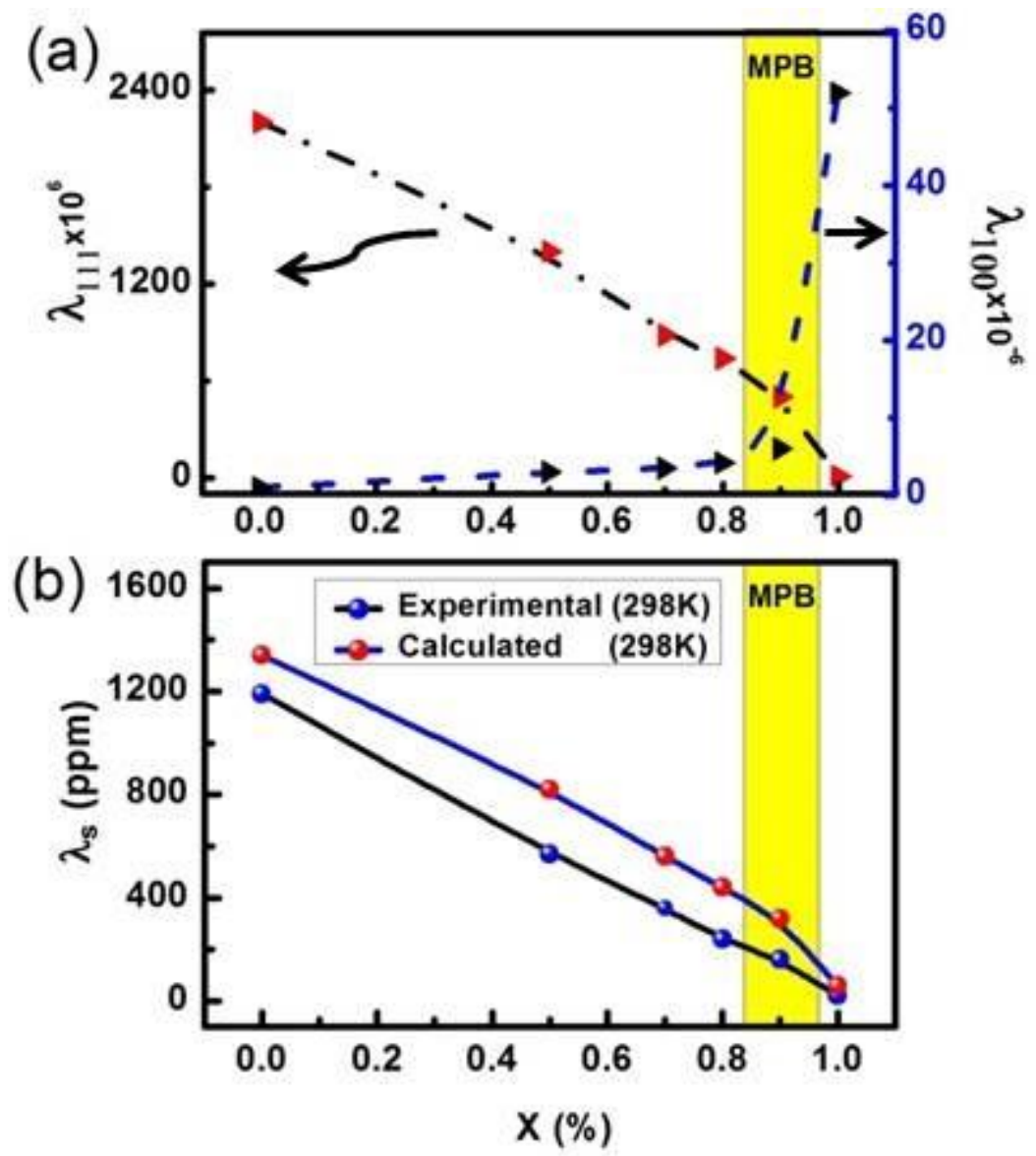


\section{References}

[1] B. Jaffe, W. R. Cook, and H. Jaffe, Piezoelectric Ceramics (Academic), New York, 1971.

[2] K. Uchino, Ferroelectric Devices (Marcel Dekker), New York, 2000.

[3] M. Ahart, M. Somayazulu, and R. E. Cohen et al, Nature (London) 451 (2008) 545.

[4] V. K. Wadhawan, Introduction to Ferroic Materials, Gordon and Breach, Amsterdam, 2000.

[5] N.A. Spaldin, [ed.] K. Rabe, C.H. Ahn and J.M. Triscone, Physics of Ferroelectrics 2007.

[6] R. E. Newnham, Acta Crystallogr. Sect. A 54 (1998) 729.

[7]A.Khachaturyan, and S. Priya, in Proceedings of the MRS Spring Meeting, San Francisco, 2013 (to be published).

[8] S. Yang, H. Bao, C. Zhou, Y. Wang, X. Ren, Y. Matsushita, Y. Katsuya, M. Tanaka, K. Kobayashi, X. Song, and J. Gao, Phys. Rev. Lett. 104 (2010) 197201.

[9] C. Zhou, S. Ren, H. Bao, S. Yang, Y. Yao, Y. Ji, X. Ren, Y. Matsushita, Y. Katsuya, M. Tanaka et al, Phys. Rev. B 89 (2014) 100101(R).

[10] M. Adil, S. Yang et.al , Appl.Phys. Lett, 106 (2015) 132403.

[11] J.R Cullen, and A. E Clark, Phys. Rev. B 15 (1977) 4510.

[12] S. Yang and X. Ren, Phys. Rev. B 77 (2008) 014407.

[13] S. Hirosawa and Y. Nakamura, J. Phys. Soc. Jpn. 51 (1982) 1162.

[14] U. Atzmony and M. P. Dariel, Phys. Rev. B 13, (1976) 4006.

[15] J. A. Chelvane. S. Kasiviswanathan, M.V. Rao, G. Markandeyulu, Bull. Mater. Sci, 27 (2004) 169-173.

[16] A.S. Salem, L.A Saleh, N.M Nayfeh. S.H Mahmood, I.Abu-Aljaraysh, J. Appl.Phys, 71 (1992) 1893-1895.

[17] D. Thoma, J. Perepezko, J. Alloys Compd, 224 (1995) 330.

[18] M. S. S. Brooks, L. Nordstrom, and B. Johansson, Physica (Utrecht) 172B (1991) 95.

[19] Baudelet et al., Europhys. Lett, 13 (1990) 751-757.

[20] Y.Ahmadizadeh, V. Soti, B and R. Abedi, Adv.Studies Theor. Phys, 37 (2009) 265271.F.

[21] C. Meyer, F. Hartmann-Boutron, Y.Gros, Y. Berthier, J.L Buevoz, J. Physique, 42 (1981) 605.

[22] N.H Duc, M.Tanm, N.D Tan, D. Givord, J.Teillet, J. Magn. Magn. Mater. 1107 (1998) 177-181.

[23] K.H.J Buschow, in Ferromagnetic Materials, edited by E.P. Wohlfarth ,NorthHolland, 1980, pp. 297.

[24] E. Burzo, and Z. Amgew, Physik. 127 (1971) 32.

[25] M. J. Besnus, A. Herr and G Fisher, J. Phys. F 9 (1971) 745.

[26] K.H.J. Buschow and R. P Van Stapele, J.Appl.Phys. 41 (1970) 4066.

[27] A. R. Piercy and K. N. R Taylor, J. Phys. C 7 (1968) 1112.

[28] A. E Clarck. Proc. 19th Conf. on Meg. \& Magnetic Materials, AIP Conf. Proceedings No. 18 (1976) 1015. 
[29] A. E Clarck, J. R Cullen, and K .Sato, Proc. $20^{\text {th }}$ Conf. on Magnetism and Magnetic Materials, AlP Conf. Proc No. 24 (1975) 670.

[30] R.Z. Levitin, A.S. Markosyan, J. Magn. Magn. Mater. 84 (1990) 247.

[31] E. Gratz, Solid State Commun. 48 (1983) 825.

[32] N. Yoshimoto, J. Sakurai, Y. Komura, J. Magn. Magn. Mater. 31-34 (1983) 137.

[33] D. Gignoux, F. Givord, R. Perrier de la Bathie, F. Sayetat, J. Phys. F: Metal Phys. 9 (1979) 763.

[34] V. Goldberg-Murmis, U. Atzmony, M.P. Dariel, J. Mater. Sci. 127-130 (1980) 127.

[35] A. E. Clark, in Ferromagnetic Materials, edited by E. P. Wohlfart, North-Holland, Amsterdam, 1980, pp. 531.

[36] A. E. Clarck, J. R. Cullen, O. D. Mcmasters and E. R. Callen, AIP Conference Proceedings 29, (1976) 192.

[37] V.V. Aleksandryan, R.Z. Levitin, A.S. Markosyan, V.V. Snegirev, A.D. Shchurova, Sov. Phys. JETP 65 (1987) 502.

[38] B. Barbara, J. P. Giraud, J. Laforest, R. Lemaire, E. Siaud, and J. Schweizer, Physica B 86-88 (1977) 155. 